\title{
Stripe sensor tomography
}

\author{
Mladen Barbic, ${ }^{1, a)}$ Lvcian Vltava, ${ }^{1}$ Christopher P. Barrett, ${ }^{1}$ Teresa H. Emery, ${ }^{2}$ and \\ Axel Scherer ${ }^{2}$ \\ ${ }^{1}$ Department of Physics and Astronomy, California State University Long Beach, 1250 Bellflower Boulevard, \\ Long Beach, California 90840, USA \\ ${ }^{2}$ Department of Electrical Engineering, California Institute of Technology, 1200 E. California Boulevard, \\ Pasadena, California 91125, USA
}

(Received 26 October 2007; accepted 18 February 2008; published online 12 March 2008)

\begin{abstract}
We introduce a general concept of tomographic imaging for the case of an imaging sensor that has a stripelike shape. We first show that there is no difference, in principle, between two-dimensional tomography using conventional electromagnetic or particle radiation and tomography where a stripe sensor is mechanically scanned over a sample at a sequence of different angles. For a single stripe detector imaging, linear motion and angular rotation are required. We experimentally demonstrate single stripe sensor imaging principle using an elongated inductive coil detector. By utilizing an array of parallel stripe sensors that can be individually addressed, two-dimensional imaging can be performed with rotation only, eliminating the requirement for linear motion, as we also experimentally demonstrate with parallel coil array. We conclude that imaging with a stripe-type sensor of particular width and thickness (where the width is much larger than the thickness) is resolution limited only by the thickness (smaller parameter) of the sensor. We give examples of multiple sensor families where this imaging technique may be beneficial such as magnetoresistive, inductive, superconducting quantum interference device, and Hall effect sensors, and, in particular, discuss the possibilities of the technique in the field of magnetic resonance imaging. () 2008 American Institute of Physics. [DOI: 10.1063/1.2894330]
\end{abstract}

\section{INTRODUCTION}

Imaging of samples through projections ${ }^{1}$ has been an important concept ever since the discovery of $\mathrm{x}$-rays, ${ }^{2}$ and has been used in the gravitational theory ${ }^{3}$ and radio astronomy ${ }^{4}$ before becoming widespread through computerized tomography ${ }^{5}$ in x-ray, ${ }^{6}$ electron, ${ }^{7}$ and optical ${ }^{8}$ imaging, among others. We also note that the first report of magnetic resonance imaging (MRI) had its roots in image reconstruction from projections. ${ }^{9}$ Computerized tomographic image reconstruction algorithms for conventional radiation-based tomography are by now well developed ${ }^{10-14}$ and can be easily transferred to novel imaging methodologies such as the one described in this article on stripe sensor tomography.

Our motivation in this work stems from the various disciplines of scanning probe microscopy where a point-bypoint raster scanning of the sample in the $x-y$ plane is a common way of obtaining a two-dimensional (2D) image of the sample. It can frequently be the case, however, that the scanning sensor is not of the point type, but is of the stripe shape, typically due to the thin film lithographic character of sensor fabrication. For example, in the scanning magnetoresistance microscopy, ${ }^{15}$ the imaging sensor is a thin film magnetoresistive element of small thickness $t$ and much larger width $w$. By raster scanning of this sensor in the $x-y$ plane, a 2D image of a magnetic sample can be obtained. ${ }^{15-18}$ There has been a perceived notion in those reports that $2 \mathrm{D}$ images obtained with a stripe-type magnetoresistive sensor are lim-

${ }^{a)}$ Electronic mail: mbarbic@csulb.edu. ited in spatial resolution by thickness $t$ in the $x$ direction, and width $w$ in the $y$ direction. It is the goal of this article to show that by linear scanning motion of the stripe-type sensor at different angular orientations combined with the tomographic imaging principles, 2D images of samples can be obtained that are limited in spatial resolution along both $x$ and $y$ axes by the thickness $t$, i.e., the smaller parameter of the sensor only.

\section{SINGLE STRIPE SENSOR IMAGING}

We first show through Fig. 1 that there is, in principle, no difference between conventional radiation-based twodimensional tomography and tomography where a stripe sensor is mechanically scanned over a sample at a sequence of different angles. Figure 1(a) shows the schematic representation of conventional tomography configuration with the parameters used in the image reconstruction process indicated. Uniformly separated parallel rays (electromagnetic or particle) from a source are used to obtain an image projection along an axis at a detector on the opposite side of the source. At each projection, the one-dimensional Radon transform of the sample density function $\rho(x, y)$ is formed:

$$
P_{\phi}(q)=\int_{(\phi, q) \text { line }} \rho(x, y) d s .
$$

By obtaining multiple one-dimensional Radon transforms (1) at different angles $\Phi$, image reconstruction is performed by the Fourier transform filtered back-projection algorithm for parallel projections: ${ }^{12}$ 

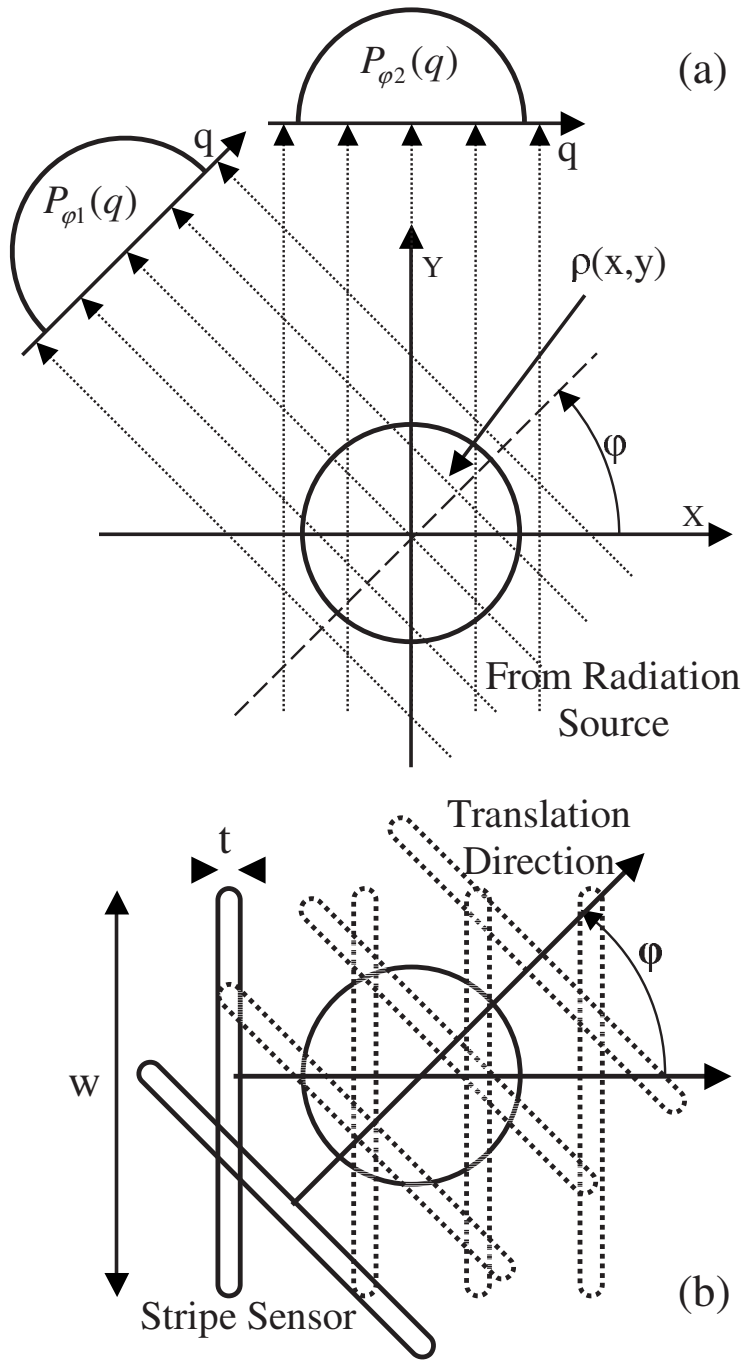

FIG. 1. (a) Conventional computerized tomography configuration. Parallel rays (electromagnetic or particle) from a source are used to obtain an image projection along an axis at a detector on the opposite side of the source. By obtaining multiple projections at different angles $\Phi$, image reconstruction is performed by the Fourier transform filtered back-projection algorithm. (b) Stripe sensor tomography where a sensor of width $w$ and thickness $t$ is mechanically scanned over a sample. Single line scan results in a onedimensional projection of a two-dimensional sample. By scanning the sensor at different angles $\Phi$ (or by rotating the sample by angle $\Phi$ and scanning the sensor along the same line), multiple projections are obtained. The same Fourier transform filtered back-projection algorithm is used for image reconstruction.

$$
\begin{aligned}
\rho(x, y)= & \int_{0}^{\pi}\left\{\int_{-\infty}^{+\infty}\left[\int_{-\infty}^{+\infty} P_{\phi}(q) e^{i 2 \pi k q} d q\right]\right. \\
& \left.\times|k| e^{-i 2 \pi k q} d k\right\} d \phi .
\end{aligned}
$$

This reconstruction process involves calculation of the Fourier transform of the Radon transform (innermost bracketed term), multiplication by a ramp function $|k|$ in conjugate space followed by an inverse transformation (outer bracketed term), and finally, integration over all angles for the completion of the image reconstruction (outermost integration term). ${ }^{12}$

Figure 1(b) shows the principle of stripe sensor tomography. A linear sensor of width $w$ and thickness $t$ is mechani-
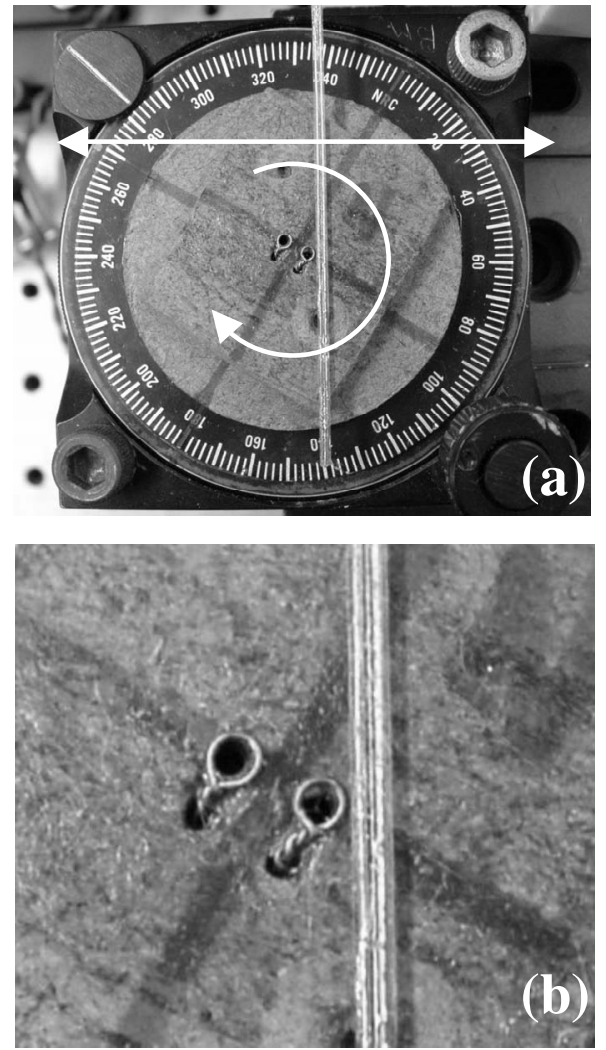

FIG. 2. (a) Experimental example for stripe sensor tomography. An elongated inductive coil loop detector is made from two parallel conductors inserted into capillary tubes for insulation and uniform separation. The loop is mounted on a linear mechanical translation stage with the axis of the linear motion indicated. The sample is a set of two circular coils, separated by a distance slightly larger than the thickness $t$ of the sensor but much smaller than the width $w$ of the sensor. The sample is mounted on the mechanical rotation stage visible in the figure. The magnified view of the two loop sources and the two conductors of the inductive stripelike coil detector are shown in (b).

cally scanned over a sample that extends over a region that is smaller than the width of the stripe sensor, as shown in Fig. 1(b). If the sensor has equal sensitivity along its width $w$ (as is assumed in this article), a single line scan of the sensor will result in the one-dimensional data that represents a onedimensional projection of the two-dimensional sample. By scanning the sensor at different angles $\Phi$ as shown in Fig. 1(b) (or by rotating the sample by angle $\Phi$ and scanning the sensor along the same line), multiple one-dimensional (1D) projections of the 2D sample represented by Eq. (1) are obtained. Finally, by utilizing the reconstruction procedure of Eq. (2), 2D imaging of the sample can be performed as we show in the next section.

Our prototypical experimental example is an elongated inductive coil loop detector, shown in Fig. 2(a). We chose this example due to its simplicity and its potential use in single sided magnetic resonance imaging ${ }^{19}$ or eddy current nondestructive evaluation. The coil is made from two parallel conductors inserted into capillary tubes for insulation and uniform separation of $t=750 \mu \mathrm{m}$. The loop is mounted on a linear mechanical translation stage with the axis of the linear motion indicated in the figure. Our "sample" is a circular coil pair, each $1.5 \mathrm{~mm}$ in diameter, representing two point sources separated by a distance slightly larger than the thick- 

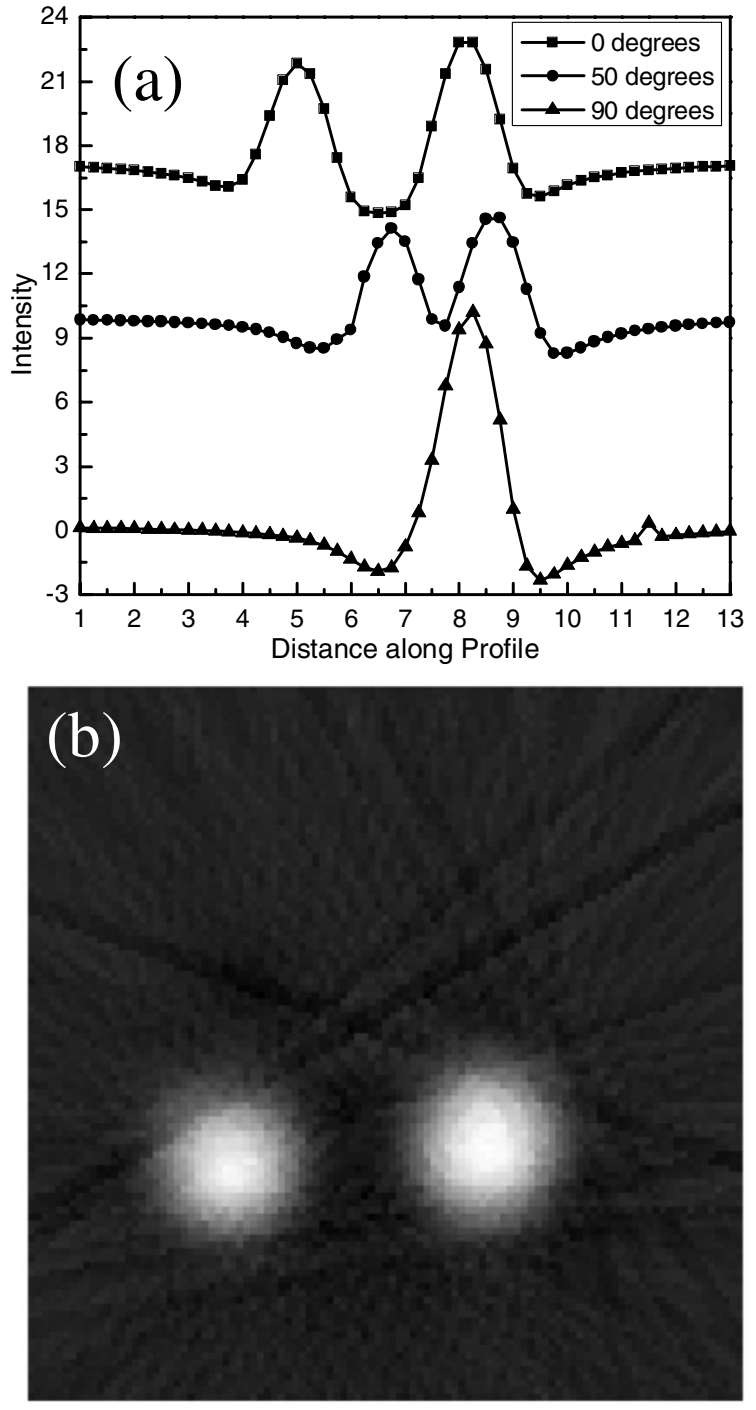

FIG. 3. Three example line scans (out of 18) representing three different 1D projections of a 2D sample at different angular orientations. (b) 2D image of the sample obtained through standard filtered back-projection image reconstruction.

ness $t$ of the sensor but much smaller than the width $w$ of the sensor. The sample is mounted on the mechanical rotation stage visible in the figure. The magnified view of the two loop sources and the two conductors of the inductive stripelike coil detector are shown in Fig. 2(b). The sources are driven in phase with $100 \mathrm{~mA}$ ac electric current at $11 \mathrm{kHz}$ from an audio power amplifier (Teach Spin model PAA1-A) driven by a lock-in amplifier signal source (Stanford Research Systems model SR830). Due to the low output impedance of the stripe coil sensor, the detected signal is coupled to a low-noise transformer preamplifier (Stanford Research Systems model SR554) followed by the signal input channel of the lock-in amplifier (Stanford Research Systems model SR830). The loop is scanned in $250 \mu \mathrm{m}$ steps at $500 \mu \mathrm{m}$ height above the surface, and the rotation stage is rotated by $10^{\circ}$ after each linear scan.

Figure 3(a) shows three example line scans (out of 18) representing three different $1 \mathrm{D}$ projections of a $2 \mathrm{D}$ sample at different angular orientations. At $0^{\circ}$, the two sources are

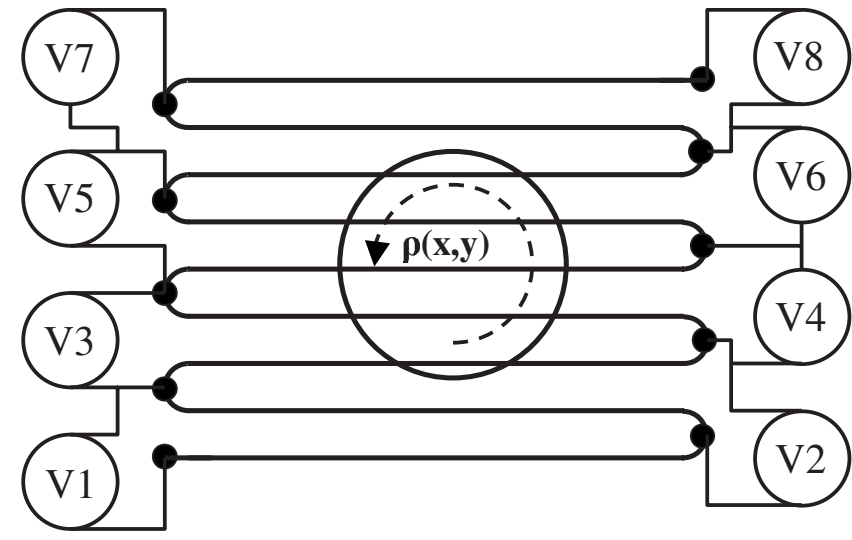

FIG. 4. Stripe sensor array tomographic imaging. By creating a meanderlike loop array and detecting voltages at sequential nodes in the array, signals are obtained without the requirement for linear translation of the sample or the array. Additionally, a single wire in the array is used in two detector loops, which minimizes the number of required wires, improves the imaging resolution, and simplifies the setup.

clearly resolved, and at $90^{\circ}$, only a single large peak is detected, as is expected for two in-phase ac sources concurrently under the detector. Figure 3(b) shows the resulting 2D image of the sample (from 18 projections) using the formalism of tomographic filtered back-projection image reconstruction of Eq. (2). The two sources are clearly resolved, and the main argument that the 2D image resolution is limited in both directions by the narrow thickness parameter only of the sensor is demonstrated.

We note the unique feature of this technique, as related to potential use in MRI, is that the imaging could be performed without the need for external high power gradient magnetic fields typically employed in MRI. In the stripe sensor case, the imaging resolution would be determined by the sensor thickness, and not by the gradient field values that could be achieved from external current carrying conductors.

\section{STRIPE SENSOR ARRAY IMAGING}

The principle of stripe-type sensor 2D tomography can be extended to the $2 \mathrm{D}$ imaging with a sensor array. The method is diagrammatically described in Fig. 4, again for the case of the stripe-type inductive detector loops. By creating a meanderlike loop array placed over the sample, and detecting voltages at sequential nodes in the array as shown in the figure, signals are obtained without the requirement for linear translation of the sample or the array. One advantage of the inductive array, constructed as shown in the figure, is that a single wire in the array is used in two detector loops. For example, the second wire from the bottom in the figure is part of the loop sensing V1 and part of the loop sensing V2. This arrangement minimizes the number of wires required in the array, improves the imaging resolution, simplifies the setup, and potentially reduces the imaging time.

In order to experimentally demonstrate this imaging mode, we prepared the sensor array, shown in Fig. 5(a), by conventional optical lithography. $50 \mathrm{Cr} / \mathrm{Au}$ wires that are $50 \mu \mathrm{m}$ wide, $100 \mathrm{~nm}$ thick, and separated by a center-tocenter distance of $400 \mu \mathrm{m}$ on a glass substrate for 50 detection loops. Wider wires connect to the array and fan out for 

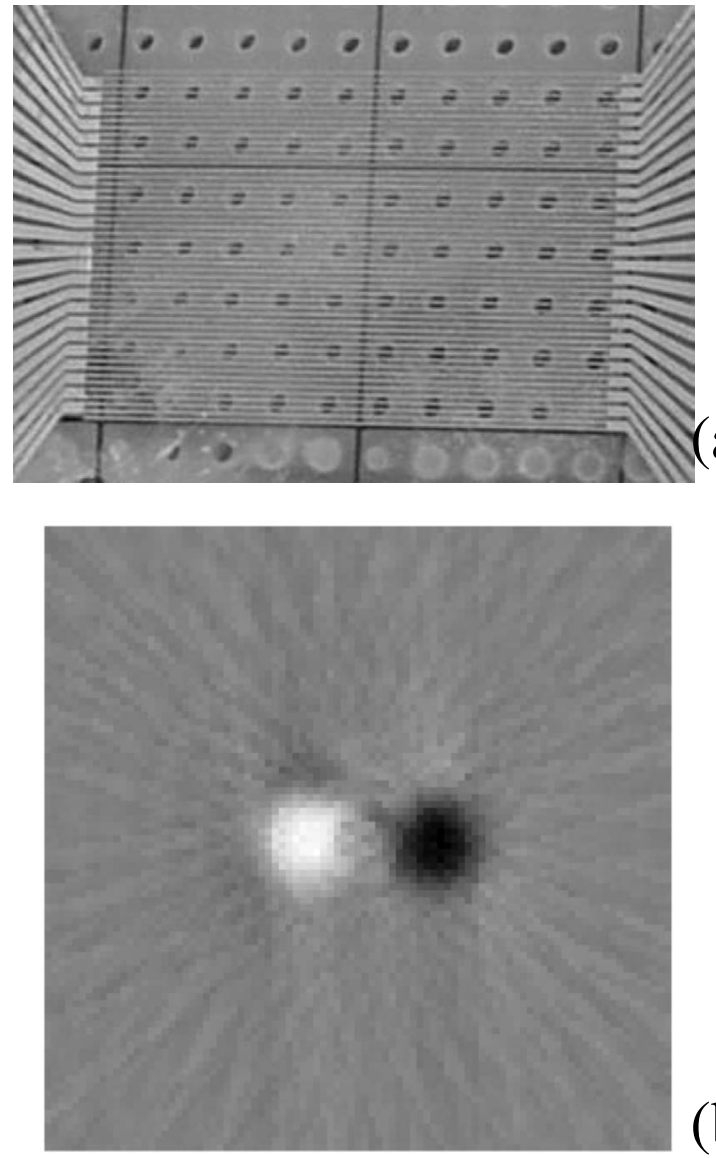

(b)

FIG. 5. Stripe sensor array fabricated by conventional optical lithography. Wider wires connect to the array and fan out for contact access. The sample is a pair of two circular coils with $180^{\circ}$ out of phase electric ac currents. The data are first collected sequentially from each loop of the array without any linear translation. The sample is then rotated by $10^{\circ}$, and procedure repeated. (b) The resulting 2D image, reconstructed using the standard filtered back-projection algorithm.

easy contact access. Our sample is again a two point circular coil pair mounted on a rotation stage, and this time electrically arranged so that the ac currents in them are $180^{\circ}$ out of phase. The data are collected sequentially from each loop of the array using the same source/audio power amplifier and transformer preamplifier/lock-in amplifier arrangement, but this time without any linear translation. After all the loop voltage signals are recorded in sequence for a single angle, the sample is rotated by $10^{\circ}$, and procedure repeated. The resulting image, reconstructed using the algorithm of Eq. (2), is shown in Fig. 5(b), again demonstrating imaging of the two point sources with $2 \mathrm{D}$ resolution limited only by the narrow parameter of the sensor.

\section{STRIPE VERSUS POINT SENSOR IMAGING}

In this section, we discuss some of the differences and consequences of stripe-type sensor imaging of Fig. 1(b), as compared to the raster scanning point probe imaging shown in Fig. 6(a). Although both imaging methodologies, the point-by-point imaging and stripe sensor imaging, provide two-dimensional images with resolution limited by the smaller parameter of the sensor (thickness of the stripe sensor and size of the point sensor, respectively), there are sev-

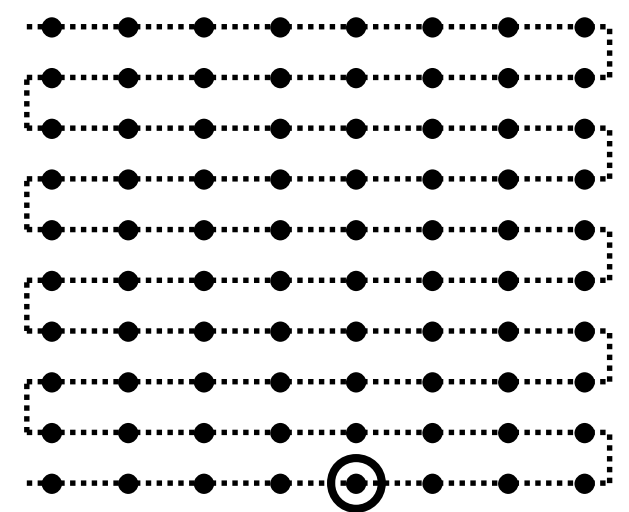

Point Sensor

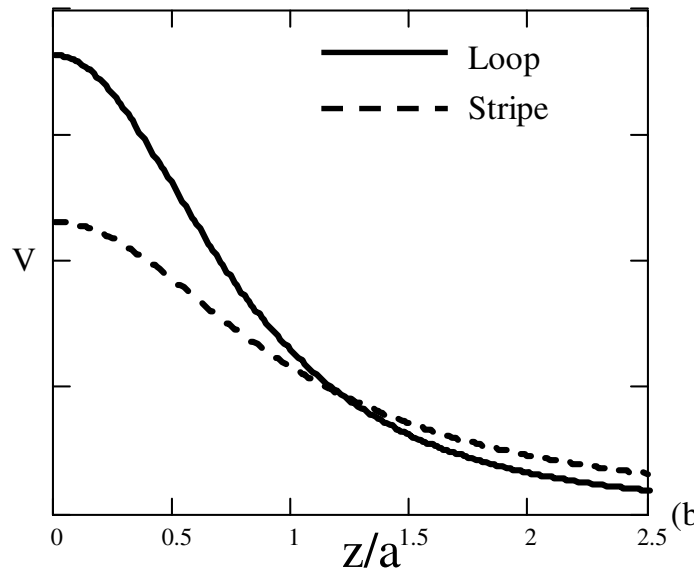

(b)

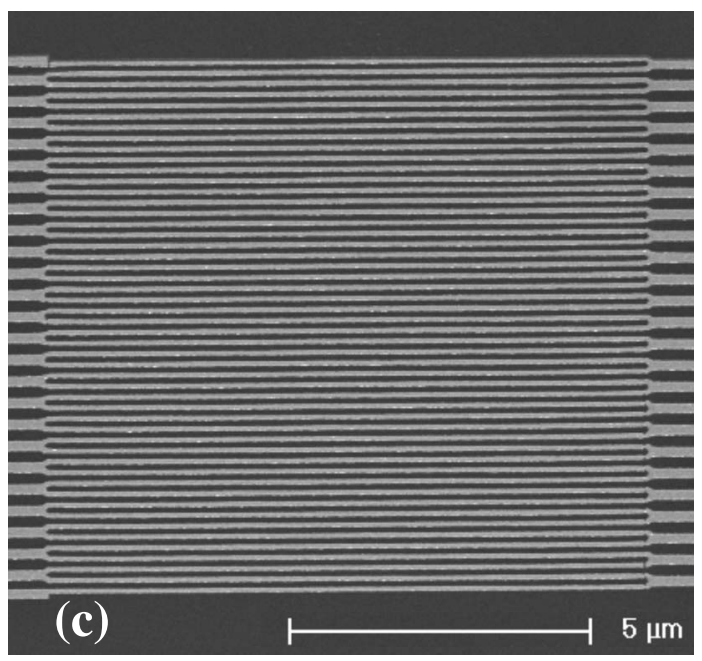

FIG. 6. (a) Raster scanning point probe $2 \mathrm{D}$ imaging. The point probe measures and resolves, for a certain measurement time $T$, each pixel of the image individually and only once during the course of the $\mathrm{N} \times \mathrm{N}$ steps imaging sequence. (b) Sensor sensitivity functions for the case of an inductive point loop and an inductive stripe loop sensors. The circular loop is more sensitive for the sample region close to the sensor, while the stripe loop is more sensitive for the sample region further away from the sensor. (c) Nanofabricated version of the inductive loop sensor array.

eral differences that we note. We focus on the inductive pickup loop detector, although similar analysis may be applied to other sensor types.

The first distinction is in the measurement time and consequently the signal-to-noise ratio of the two methodologies. In the raster scanning point probe method of Fig. 6(a), the point probe measures and resolves for a certain measurement time $T$ each pixel of the image individually and only once 
during the course of the $N \times N$ step imaging sequence. In contrast, for the stripe sensor imaging mode of Fig. 1(b), the sensor detects multiple pixels of the image for a certain measurement time $T$ at each step of the $N$ steps linear scan and does not resolve the pixels along the sensor width. Nevertheless, through $M$ angular orientations of the linear scans, as we described in this article, it still obtains the 2D imaging with resolution defined by the thickness of the sensor only. However, note that each pixel of the image in the stripe sensor imaging method is detected by the sensor for every angular orientation of the linear scan, and is therefore detected $M$ times. How this affects the signal to noise ratio (SNR) is difficult to precisely estimate. $M$ times more measurements of each image pixel in the stripe sensor technique should provide $\sqrt{M}$ times better SNR, but the larger length of the striped-shaped inductive loop sensor results in the larger sensor resistance $R$, and therefore larger rms noise of the sensor which scales as $\sqrt{R}$.

There is also a slight difference in the depth of view between the two imaging modalities for the case of an inductive sensor. Using the principle of reciprocity, ${ }^{20}$ often used in the theory and practice of magnetic resonance ${ }^{21,22}$ and magnetic recording ${ }^{23}$ for signal reception analysis, we can compare the field patterns of the two methods and estimate the relative $z$-axis dependence of sensitivity between the two sensor shapes. Figure 6(b) shows the difference between the sensor sensitivity as a function of the sample distance along the $z$ axis (perpendicular to the sample surface). For the stripe sensor with wires of the stripe loop separated by distance $2 a$ as compared to the circular loop of radius $a$, the graph indicates that the circular loop is more sensitive for the region of the sample close to the sensor, while the stripe loop is more sensitive for the region of the sample that is further away from the sensor. Therefore, the stripe sensor has a larger "depth of view" and weaker depth dependence of the received signal, while the point sensor is more sensitive at the surface of the sample and has steeper depth dependence of reception. The crossover point for sensitivity is at $z=1.21 \mathrm{a}$. We note that, as related to potential use in MRI, a vertical field gradient could be employed for extending the imaging process to three dimension. However, the signal from spins that are over a given stripe detector in the array start to significantly contribute to the signals being detected by neighboring stripe detectors. Therefore, spatial resolution will suffer further away from the detector array.

One can also make a comparison between the N-stripe array sensor described in Figs. 4 and 5 and the $N \times N$ point sensors array used, for example, in the detection of biomedically related magnetic fields. ${ }^{24}$ Again, both methods, in principle, achieve similar 2D spatial imaging resolution. The stripe sensor array needs $\mathrm{N}$ times fewer sensors but requires sample (or array) rotation and tomographic computer reconstruction, while the $N \times N$ point sensor array does not require mechanical motion, but requires $\mathrm{N}$ times more detection channels for 2D image acquisition.

There are two additional challenges that are present in the stripe sensor tomography that we mention. Flat surface of the sample is one major restriction. This limitation is typically not an issue in point sensor scanning probe microscopy, where surface topography is obtained along with any other parameter of the sample (electrostatic, magnetic, etc.). Additionally, stripe sensor tomography requires the uniformity of sensor sensitivity response along the width of the sensor. This is well satisfied for an inductive sensor we presented, but could be more problematic in, for example, magnetoresistive stripe sensor, where slight variation in the point sensitivity function along the sensor length has been experimentally observed. ${ }^{25,26}$

\section{POTENTIAL APPLICATIONS}

We mention that there are many other sensor families that may be suitable for stripe sensor tomographic implementation. We list thin film magneto-resistive sensors, ${ }^{15-18}$ asymmetric superconducting quantum interference devices (SQUIDs), ${ }^{27}$ elongated microfabricated waveguides, ${ }^{28}$ striplines, ${ }^{29}$ and nanoparticle-tape-filled microcoils ${ }^{30}$ used in NMR detection, planar asymmetric micro-Hall detectors, ${ }^{31}$ and microwave near-field slit probes ${ }^{32,33}$ as suitable candidates. Another possibility is the extension of the stripe array sensor idea into the submicron MRI resolution regime.

In Fig. 6(c), we show a nanofabricated version of the inductive loop sensor array. In this regime, the flatness of the sample and the requirement for angular rotation of the sample may prove especially difficult. We suggest that electrorotation $^{34}$ of samples may provide viable solution to the nanoscale angular orientation challenge. Although such miniaturized conductor structures have increased resistance and therefore degrade SNR of an inductive detector, they also provide a higher field per unit current, $B_{1} / I$, at the sample location. ${ }^{21,22}$ Careful and extensive analysis of SNR of microcoil structures in NMR detection ${ }^{35,36}$ indicates that SNR per unit volume increases as the inductive detector decreases in size, further motivating size reduction of the inductive stripe sensor array. It is also interesting to point out that in potential magnetic resonance imaging implementation on this size scale, the technique would operate in the regime where nuclear spin noise signal is appreciable and comparable to the conventional NMR signal. ${ }^{37}$ Therefore, 2D imaging with a submicron stripe sensor array could in that case be performed without the need for external imaging gradient fields and without the need for high power radio-frequency excitation. We find this possibility especially attractive and worthy of further pursuit.

\section{ACKNOWLEDGMENTS}

This material was based upon work supported by the National Science Foundation under the NSF-CAREER Award Grant No. 0349319, NSF-ECS Award Grant No. 0622228, NIH Award No. R01-HG0026440, as well as the California State University Long Beach Scholarly and Creative Activities Award (SCAC).

\footnotetext{
${ }^{1}$ S. Webb, From the Watching of Shadows: The Origins of Radiological Tomography (Hilger, New York, 1990).

${ }^{2}$ W. C. Röntgen, Nature (London) 53, 274 (1896).

${ }^{3}$ J. Radon, Ber. Verh. Saechs. Akad. Wiss. Leipzig, Math.-Phys. K1. 69, 262 (1917).

${ }^{4}$ R. N. Bracewell, Aust. J. Phys. 9, 198 (1956).
} 
${ }^{5}$ A. Cormack, J. Appl. Phys. 34, 2722 (1963).

${ }^{6}$ G. Hounsfield, Br. J. Radiol. 46, 1016 (1973).

${ }^{7}$ D. J. de Rosier and A. Klug, Nature (London) 217, 130 (1968).

${ }^{8}$ D. E. Kuhl and R. Q. Edwards, Radiology 80, 653 (1963).

${ }^{9}$ P. C. Lauterbur, Nature (London) 242, 190 (1973).

${ }^{10}$ G. T. Herman, Image Reconstruction from Projections (Academic, New York, 1980).

${ }^{11}$ F. Natterer, The Mathematics of Computerized Tomography (Wiley, New York, 1986).

${ }^{12}$ A. C. Kak and M. Slaney, Principles of Computerized Tomographic Imaging (SIAM, Philadelphia, 2001).

${ }^{13}$ S. R. Deans, The Radon Transform and Some of Its Applications (Krieger, Malabar, 1993).

${ }^{14}$ P. T. Callaghan, Principles of Nuclear Magnetic Resonance Microscopy (Oxford University Press, New York, 1991).

${ }^{15}$ S. Y. Yamamoto and S. Schultz, Appl. Phys. Lett. 69, 3263 (1996).

${ }^{16}$ S. Y. Yamamoto, R. O'Barr, S. Schultz, and A. Scherer, IEEE Trans. Magn. 33, 3016 (1997).

${ }^{17}$ M. Todorovic, S. Schultz, J. Wong, and A. Scherer, Appl. Phys. Lett. 74, 2516 (1999).

${ }^{18}$ M. Barbic, S. Schultz, J. Wong, and A. Scherer, IEEE Trans. Magn. 37, 1657 (2001).

${ }^{19}$ B. Blumich, NMR Imaging of Materials (Oxford University Press, New York, 2000).

${ }^{20}$ W. F. Brown, Jr., Magnetostatic Principles in Ferromagnetism (North-Holland, Amsterdam, 1962).

${ }^{21}$ D. I. Hoult and R. E. Richards, J. Magn. Reson. 24, 71 (1976).
${ }^{22}$ D. I. Hoult and P. C. Lauterbur, J. Magn. Reson. 34, 425 (1979).

${ }^{23} \mathrm{~S}$. X. Wang and A. M. Taratorin, Magnetic Information Storage Technology (Academic, San Diego, 1999).

${ }^{24}$ The SQUID Handbook, edited by J. Clarke and A. I. Braginski (WileyVCH, Berlin, 2006).

${ }^{25}$ G. A. Gibson, S. Schultz, T. Carr, and T. Jagielinski, IEEE Trans. Magn. 28, 2310 (1992).

${ }^{26}$ M. Todorovic and S. Schultz, J. Appl. Phys. 83, 6229 (1998).

${ }^{27}$ S.-J. Kim, J. Chen, K. Nakajima, T. Yamashita, S. Takahashi, and T. Hatano, J. Appl. Phys. 91, 8495 (2002).

${ }^{28}$ Y. Maguire, I. L. Chuang, S. Zhang, and N. Gershenfeld, Proc. Natl. Acad. Sci. U.S.A. 104, 9198 (2007).

${ }^{29}$ P. J. M. van Bentum, J. W. G. Janssen, A. P. M. Kentgens, J. Bart and J. G. E. Gardeniers, J. Magn. Reson. 189, 104 (2007).

${ }^{30}$ M. Barbic and A. Scherer, Solid State Nucl. Magn. Reson. 28, 91 (2005).

${ }^{31}$ H. Guillou, A. D. Kent, G. W. Stupian, and M. S. Leung, J. Appl. Phys. 93, 2746 (2003).

${ }^{32}$ F. Sakran, A. Copty, M. Golosovsky, N. Bontemps, D. Davidov, and A. Frankel, Appl. Phys. Lett. 82, 1479 (2003).

${ }^{33}$ F. Sakran, A. Copty, M. Golosovsky, D. Davidov, and P. Monod, Appl. Phys. Lett. 84, 4499 (2004).

${ }^{34}$ W. M. Arnold and U. Zimmermann, J. Electrost. 21, 151 (1988).

${ }^{35}$ T. L. Peck, R. L. Magin, and P. C. Lauterbur, J. Magn. Reson., Ser. B 108, 114 (1995).

${ }^{36}$ A. G. Webb, Prog. Nucl. Magn. Reson. Spectrosc. 31, 1 (1997).

${ }^{37}$ N. Muller and A. Jerschow, Proc. Natl. Acad. Sci. U.S.A. 103, 6790 (2006). 